\title{
f Theme: Infectious Diseases
}

SARS-CoV-2 Infection among community health workers in India before and after use of face shields ( $J A M A$. Published online August 17, 2020)

As the SARS-CoV-2 pandemic progresses relentlessly, it is believed that close proximity with airborne respiratory droplets from infected persons is responsible for disease transmission. This study attempted to look at the difference in transmission if any, before and after the use of face shields.

The study was commenced on May 3, 2020 on the community health care workers of a research network at Chennai, India. They were assigned for counselling of asymptomatic family contacts of patients who had tested positive for SARS-CoV-2 at their residence. They maintained social distancing at all times and wore 3-layered surgical masks, gloves and shoe covers, and used hand rub.

Two workers became symptomatic 2 weeks later. The remaining 60 workers were tested for SARS-CoV-2 by RT-PCR. After this, the workers were provided with face shields of $250 \mu \mathrm{m}$ thickness made of polyethylene terephthalate which were decontaminated at the end of the day. The workers were screened weekly for symptoms and RT-PCR

Comparison between the positive test results before and after the introduction of face shields was done. After the introduction of the face shields, 50 previously uninfected workers continued to provide counselling and no worker developed symptomatic or asymptomatic infection. The face shields may have helped to decrease the ocular exposure, contamination of masks or hands and diversion of air movement from around the face. Some limitation of this study include the before- after design. Further investigation of face shields in community settings is warranted.

Height of fever and invasive bacterial infection (Arch Dis Child. Published Online August 20, 2020)

This study aimed at correlating the height of fever with the occurrence of invasive bacterial infection (IBI) in infants below 60 days of age. This case-control study included infants who appeared well. The maximum temperature of these infants was measured either at home or in the ER. The cohort included infants with and without invasive bacterial infection. Interval likelihood ratios for the diagnosis of invasive bacterial infection IBI was computed at intervals of half-degree Celsius. Results showed that temperatures between 39-39.9 pC was associated with a higher likelihood of IBI. However, $30.4 \%$ of infants with IBI had lower maximal temperatures at $38.5 \mathrm{pC}$. This study proved that height of temperature alone cannot be used as a risk stratification tool for IBI.

Parents' knowledge and attitudes towards the use of antibiotics in patients within the paediatric age range (Pediatr Oncall J. 2020;17)

Antibiotics play a major role in the medical practice, accounting for an increasing number of prescriptions. Antibiotic resistance is a growing menace that poses a threat to the existing drugs and prompts a need to discover newer molecules. This study has attempted to analyze the knowledge and attitudes of parents towards antibiotic prescription. Respondents were parents of children below age 18 years living in urban as well as rural regions of Lisbon, Portugal $(n=294)$. Approximately $51 \%$ understood that antibiotics were specific for treating bacterial infections, $35 \%$ thought they needed to be administered for all types of infections. While $81 \%$ acknowledged the occurrence of side effects, $31 \%$ felt they were not satisfied if antibiotics were not prescribed to them.

This study corroborates the lack of knowledge of parents in relation to the use of antibiotics. Therefore, it is important for health professionals to understand this lacuna and educate the patients, which will help in ensuring compliance of the prescribed treatment. A higher level of education and the promotion of information campaigns by the mass media could result in a greater degree of knowledge regarding antibiotic resistance and adverse effects.

Evolution and expansion of multidrug-resistant malaria in South East Asia: A genomic epidemiology study (Lancet. 2019;19:943-51)

A multidrug-resistant co-lineage of Plasmodium falciparum malaria, named KEL1/PLA1 was found in Cambodia between 2008 -2013. This was responsible for a high rate of treatment failure with the frontline combination drugs dihydroartimesinin-piperaquine.

An epidemiology study was undertaken to analyze the whole genome sequence data samples obtained from the Malaria GEN $P$. falciparum Community Project. A large proportion of the samples were collected during the clinical studies, while other unpublished sample data was retrieved from other projects. DNA from dried spot samples were subjected to whole genome amplification before sequencing. The kelch 13 mutation is known to be associated with artemisinin resistance. Sequencing was done to align with the kelch 13 amino acid positions 350 and above.

Results were analyzed from a dataset of 2465 whole parasite genome on samples collected. The study showed that the frequency of KEL1/PLA1 increased over the study period and more than half the parasites sampled in the later part of the study were KEL1/ PLA1, indicating the expansion of the co-lineage. Before 2009, the KEL1/PLA1 was found only in western Cambodia, but a rapid rise was notable in north eastern Thailand and Vietnam. This study suggests that multiple KEL1/PLA1 subgroups were able to spread rapidly across borders in separate transmission waves, following the acquisition of exclusive mutations.

This is of significance as malaria incidence and mortality has been increasing since 2015, putting the global targets of malaria control at risk. The findings show an evolutionary process in action. KEL1/PLA1 can be viewed as an aggressive cell line, invading new territories and acquiring new genetic properties. Effective longitudinal genetic surveillance is crucial to support timely decisions on first line therapy. and guide elimination efforts against multi-drug resistant $P$. falciparum.

Sumitha NaYaK snayak2007@yahoo.co.in 